\title{
Backhaul Media Solution for Improvement of Spectrum Efficiency in LTE Networks
}

\author{
Ibrahim Umar \\ Dept. of Telecomm. Engg. \\ Institute of Comm. Technology \\ Islamabad, Pakistan
}

\author{
Muhammad Waheed, PhD \\ Dept. of Telecomm. Engg. \\ Institute of Comm. Technology \\ Islamabad, Pakistan
}

\begin{abstract}
Designing of backhaul media is one of the most challenging task in implementation LTE 4G networks. Traditionally microwave/DRS of STM-1 links were used to implement LTE network. However, due to low microwave efficiency targeted bandwidth requirements of LTE were hard to achieve. Also, operators in developing countries tend to go for cost-effective solution due to lower purchase power of the people. In this research, backhaul media is examined in detail. Even though optical fiber and microwaves are used in most countries, it is not a budget friendly option for developing countries. Some developing nations still have copper wires installed in masses which are used for internet services at home/Business area. A new solution has been proposed to use copper medium as a backhaul media for LTE 4G networks. Simulations have been performed to demonstrate the effectiveness of the idea.
\end{abstract}

\section{General Terms}

Wireless Networks, LTE Telecommunication

\section{Keywords}

Long Term Evolution, LTE, 4G, Backhaul, Wireless, Network Availability

\section{INTRODUCTION}

Over the last two decades the world has seen a rapid growth in wireless networks with ever-growing advancement in technology. Due to communication demands and requirements for data communication has triggered significant development in the industry [2][3]. The continued progress has in bandwidth and QOS (Quality of Service) has resulted in evolution of $1 \mathrm{G}$ to $4 \mathrm{G}$ faster than expected, providing with higher data rates and secure communication [7],[8].

In most recent evolution of wireless networks, the infrastructure is only IP based packet solution and known as fourth generation cellular system [3][5]. LTE also belong to latest system of $3 \mathrm{GPP}$ family and referred as $3.9 \mathrm{G}$ which is pre $4 \mathrm{G}$ but beyond $3 \mathrm{G}$.

Due to increase in data rates over the generation, the backhaul media requirements are also changed. In first generation mobile networks, the maximum data rate is $1 \mathrm{~K}$ and requires a backhaul media of 2 Mbps which could easily be achieved by small capacity microwave links [9]. Nowadays, with LTE 4G with data rates up to $100 \mathrm{Mbps}$ and beyond for $20 \mathrm{MHz}$ spectrum, a backhaul media of $100 \mathrm{Mbps}$ is required [1].

Mostly researchers are trying to use optical fiber as back haul transmission media [4][9]. Where optical fiber is not available, high capacity Microwave/DRS links are used as a backhaul media [6],[9].
Designing and implementing a backhaul media for LTE networks is a challenging task and requires substantial resources [3]. In developing countries, budget restrictions are a hindrance in heavy investment in deployment of new networks (acquiring licenses and installations). Alongside budget restrictions, weather and terrain restrictions may also limit the use of Microwave/DRS as a backhaul media [1].

In Pakistan, PTA (Pakistan Telecommunication Authority) issued LTE licenses to three corporations for $10 \mathrm{MHz}$ bands. Currently, only a quarter of the mobile subscribers are availing $3 \mathrm{G} / 4 \mathrm{G}$ services, with $15.32 \%$ mobile broadband penetration [10]. The service providers opt for smaller bands keeping the customer demands in consideration.

In this paper, it is to investigate the feasibility of existing backhaul media for LTE and effective spectrum usage, where smaller wireless bandwidth is available. Also, presents to use copper wire networks as a backhaul media for wireless networks. This may be well suited for countries where existing copper network is deployed for internet and voice services and financial issues are limiting the evolution. Also, it may act as a standby where backhaul exists on microwave links or optical fiber.

\section{ANALYTICAL MODELING}

Considering the telecommunication companies in Pakistan as a test case, Study has been done on LTE spectrum channels currently used in Pakistan, which are $5 \mathrm{MHz}$ and $10 \mathrm{MHz}$. As this paper suggests the use of copper network as a backhaul media, so, use the stats of the company which have both copper network and LTE network. In Pakistan, only PTCL provides these two solutions together so taking spectrum of PTCL as a test example.

To analyze the 4G/LTE spectrum (5 MHz) for exiting backhaul media, see Fig. 1. Under ideal conditions throughput can be $36 \mathrm{Mbps}$, per user analysis show average speed for 1.2 Mbps (for 30 users).

Now let's analyze the 4G/LTE Spectrum (5 MHz) results for proposed backhaul media structure shown in Fig. 2. The previously covered area is divided in to three portions with each eNodeB covering from $250 \mathrm{~m}$ to $350 \mathrm{~m}$.

A difference can be seen of average throughput in Fig. 1 is 1.2 Mbps and in Fig. 2 is 3.6 Mbps. With the same spectrum, the average speed is increased up to three times. Although, increasing the number of BTS results in increased cost, but, the cost of additional BTS can easily be adjusted from transmission media. In the proposed architecture existing copper media is used and distribution poles of network, so, the expenses are saved by not using microwave media and civil structures (like tower/poles). Also, reduced coverage BTS are used so they result in lower per BTS cost. 


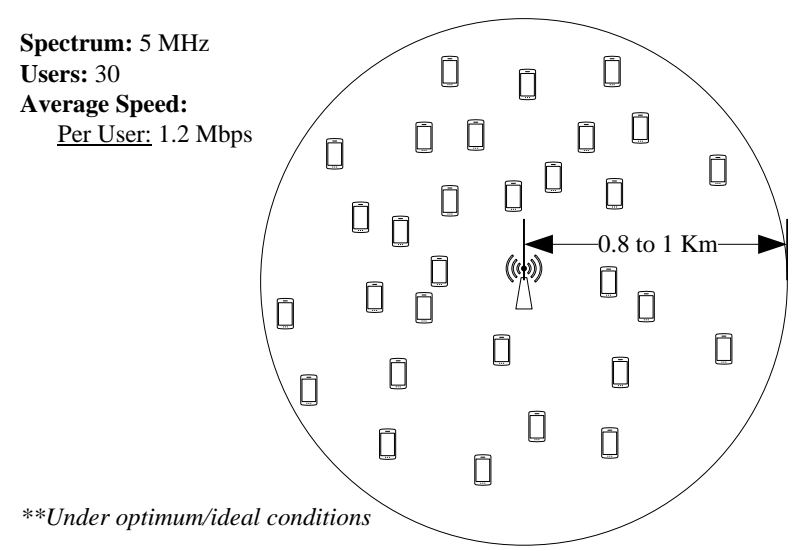

Fig. 1 - Throughput analysis for single eNodeB

This model is easy to implement with an added advantage of redundancy. In case of a BTS failure, adjacent BTS are used to compensate for loss in coverage.

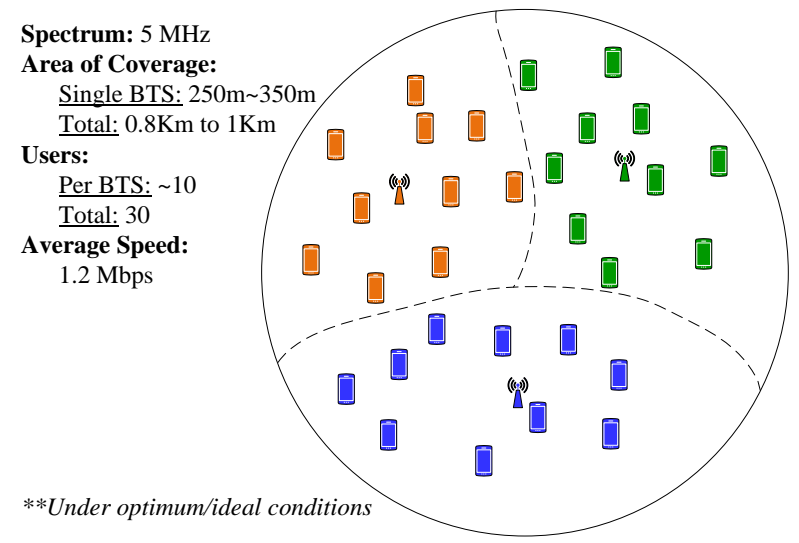

Fig. 2 - Throughput analysis for multiple eNodeB

\subsection{Network availability}

It is observed a major improvement in throughput of proposed solution over existing network. Secondary advantage of proposed solution is network availability Key Performance Indicators (KPI's).
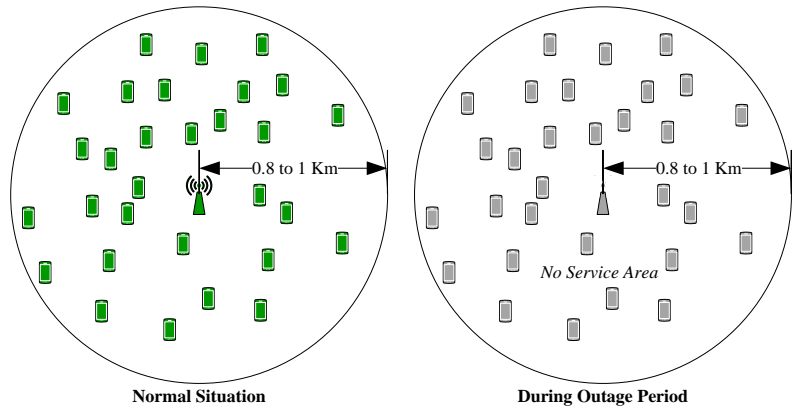

Fig. 3 - Existing Network Outage

Starting with the network availability of existing deployed structure, The scenario can be seen of single eNodeB/BTS serving an area of $0.8 \sim 1 \mathrm{Km}$ during outage period in Fig. 12. Now, in case there is an outage at this BTS due to any reason all area served from this BTS would be blacked and during shutdown period no service enable in whole area

As shown in Fig. 3 It can be say that if system is down due to some reason, the whole area served from this eNodeB will not get any service during the outage period. If a calculation has been don the network availability of single EnodB down for 24 hours:

$$
\text { Network Availability (NA) }=\frac{\left(T-t_{n o}\right)}{T} \times 100 \%
$$

Where $T$ is the total time and $t_{n o}$ is outage period. Consider the units to be minutes, If it is needed to calculate network availability during one whole day and outage was one hour.

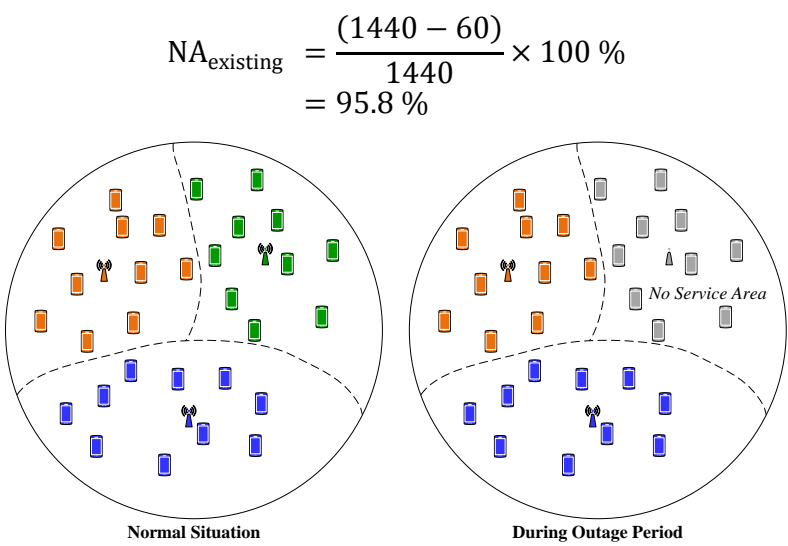

Fig. 4 - Existing Network Outage

On the other hand, if proposed solution is implemented and same issue occurs (one of the BTS fails) is shown in Fig. 4. It can be observed that during outage, approximately $66 \%$ of area is still served. Network availability for one day with one hour outage can be calculated as:

$$
\begin{aligned}
\mathrm{NA}_{\text {proposed }} & =\frac{\left(T-t_{n o} / 3\right)}{1440} \times 100 \% \\
\mathrm{NA}_{\text {proposed }} & =\frac{(1440-20)}{1440} \times 100 \% \\
& =98.6 \%
\end{aligned}
$$

So, the proposed solution is more efficient in terms of Network Availability KPI's.

\section{IMPLEMENTATION AND RESULTS}

In previous section, An ideal/optimum has been taken conditions for calculations. In this section, it can be validate the effectiveness of the proposed solution for existing and proposed methods.

\subsection{Single BTS throughput testing}

For a $5 \mathrm{MHz}$ spectrum with Backhaul Microwave media of $155 \mathrm{Mbps}$, A four-member team is made to perform the test.

\subsubsection{Single user test}

Firstly, Single user connect with BTS with $5 \mathrm{MHz}$ spectrum and the results are in Fig. 5. In this test, it is measured a maximum download speed of $30 \mathrm{Mbps}$ which is less than previously assumed 36 Mbps. Thus the actual speed a user will be getting in Fig. 1 is $1 \mathrm{Mbps}$, for 30 connected users to a single BTS. 


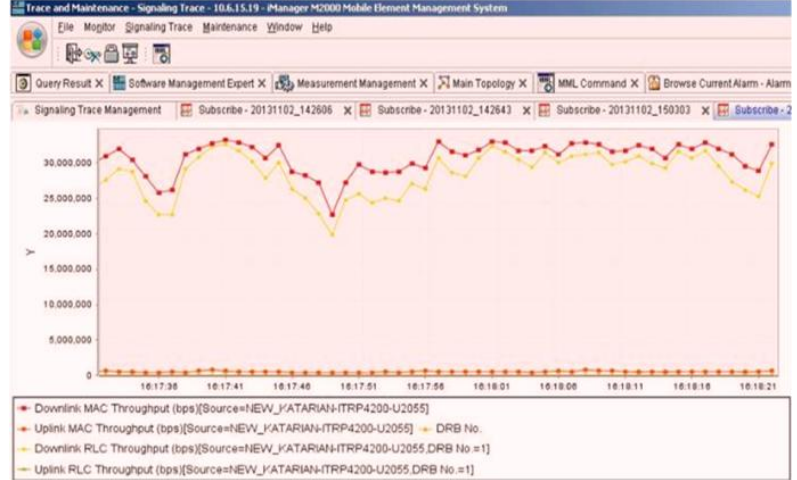

Fig. 5 - Throughput analysis in M2000

Another test is done to see how bandwidth is divided among multiple user in the same sector of the BTS. For this test, connect two, three and four users at a time on same BTS and found these results. Only test for two and four users is discussed here.

\subsubsection{Two users test}

The first user obtains an average download speed of $16 \mathrm{Mbps}$, while the second user obtains 14 Mbps, as shown in Fig. 6. Resulting in the total download speed of $30 \mathrm{Mbps}$.

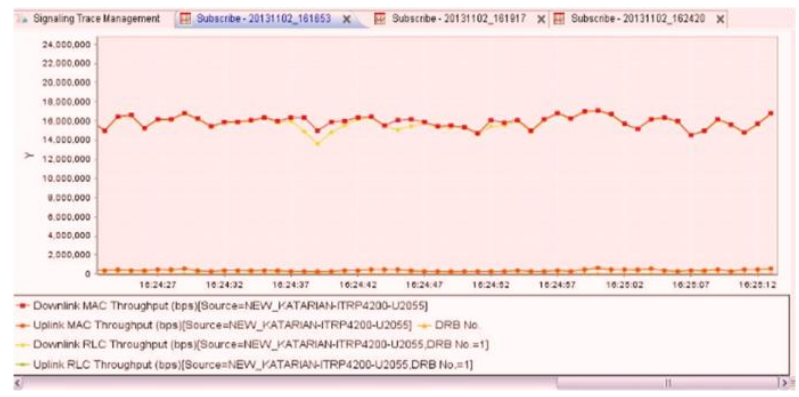

(a) - First User

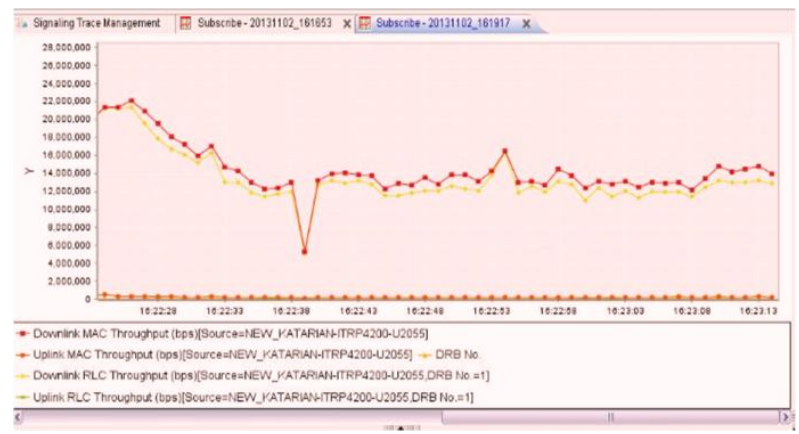

(b) - Second User

Fig 6 - Two users' throughput analysis

It is observed a marginal difference between throughputs of these two users; however, the overall throughput of eNodeB is same as previous test.

\subsubsection{Four users test}

To further verify the distribution of throughput it is to run another test in which four user connect with BTS/eNodeB. Rest conditions of this test are the same as the previous two tests.

The test results shows that that four users obtained an average download speed of $7 \mathrm{Mbps}, 8 \mathrm{Mbps}, 8 \mathrm{Mbps}$ and $7 \mathrm{Mbps}$, shown in Fig. 7 (a to d), respectively. The sum of the per user average speed is $30 \mathrm{Mbps}$. Here, it is founded that all four users have almost same throughput of 7 8 Mbps, and again the sum of all throughputs is same as the previous tests.

Table 1 shows a summary of results, A test results for three users also included. Now, it can be concluded that the maximum throughput does not exceed beyond $30 \mathrm{Mbps}$, regardless of the number of the connected users to a BTS.

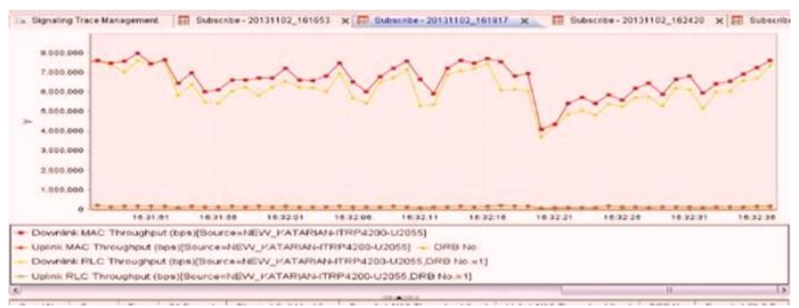

(a) - First User

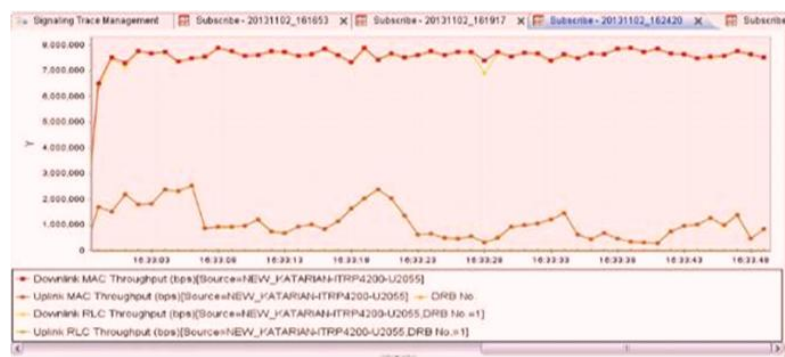

(b) - Second User

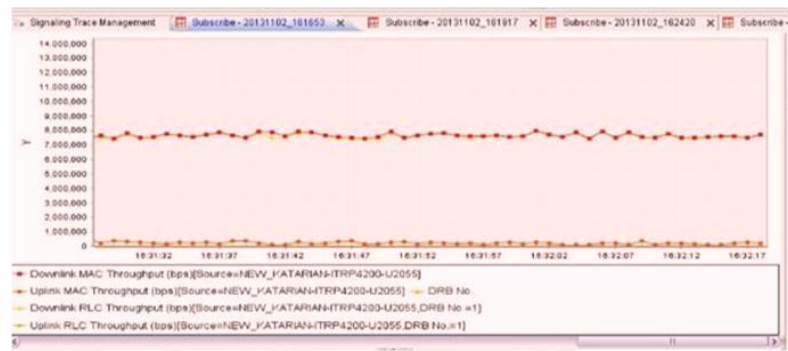

(c) - Third User

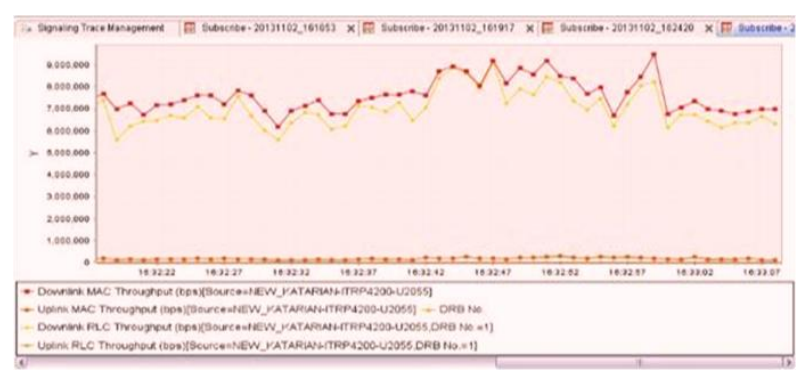

(d) - Fourth User

Fig 11 - Four users' throughput analysis

Table 1: Summary of Test Results

\begin{tabular}{|c||c|c|c|c||c|}
\hline $\begin{array}{c}\text { Total } \\
\text { Users }\end{array}$ & $\begin{array}{c}\text { User 1 } \\
\text { (Mbps) }\end{array}$ & $\begin{array}{c}\text { User 2 } \\
\text { (Mbps) }\end{array}$ & $\begin{array}{c}\text { User 3 } \\
\text { (Mbps) }\end{array}$ & $\begin{array}{c}\text { User 4 } \\
\text { (Mbps) }\end{array}$ & $\begin{array}{c}\text { Total } \\
\text { Throughput } \\
\text { (Mbps) }\end{array}$ \\
\hline 1 & 30 & Offline & Offline & Offline & 30 \\
\hline 2 & 16 & 14 & Offline & Offline & 30 \\
\hline 3 & 10 & 10 & 10 & Offline & 30 \\
\hline 4 & 7 & 8 & 8 & 7 & 30 \\
\hline
\end{tabular}




\subsection{Multiple BTS test}

To validate the effectiveness of the proposed solution, a test is performed with multiple BTS. For the test, a BTS is taken and reduced its range from $1 \mathrm{Km}$ to 500 meter and configure its backhaul media up to $55 \mathrm{Mbps}$ (achievable by copper medium). The backhaul media provides for multiple BTS in this scenario. Multiple BTS are covering the same area which was covered by a single BTS in the previous subsection.

Fig. 10 shows the combined throughput of the multiple reduced range BTS. Also, the number of connected users during the same time interval is shown in Fig 11.

The maximum Throughput was approximately $55 \mathrm{Mbps}$ and at that time 17 users were connected to the BTS. Now, if calculate average throughput for single user for, a result obtain 3.23 Mbps (55 Mbps/17).

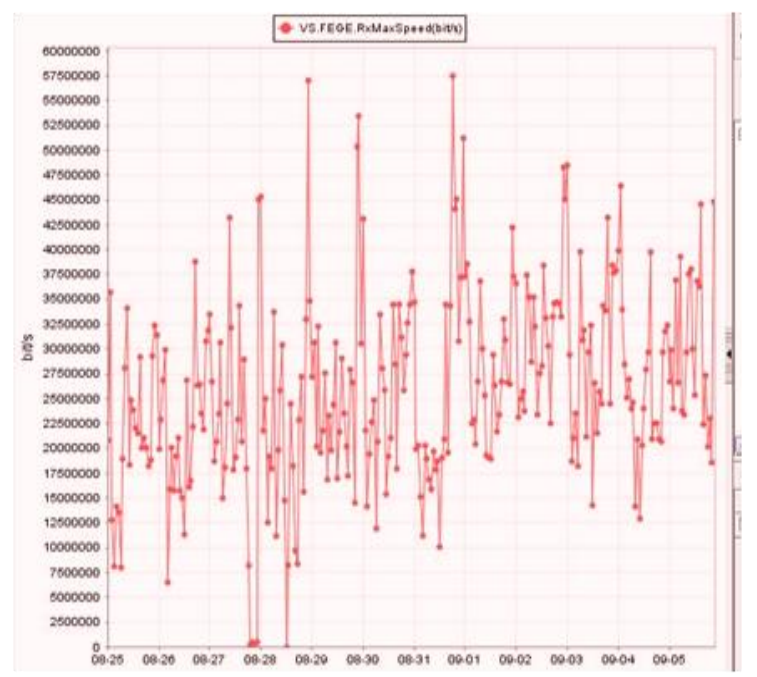

Fig 10 - Reduced range eNodeB throughput analysis

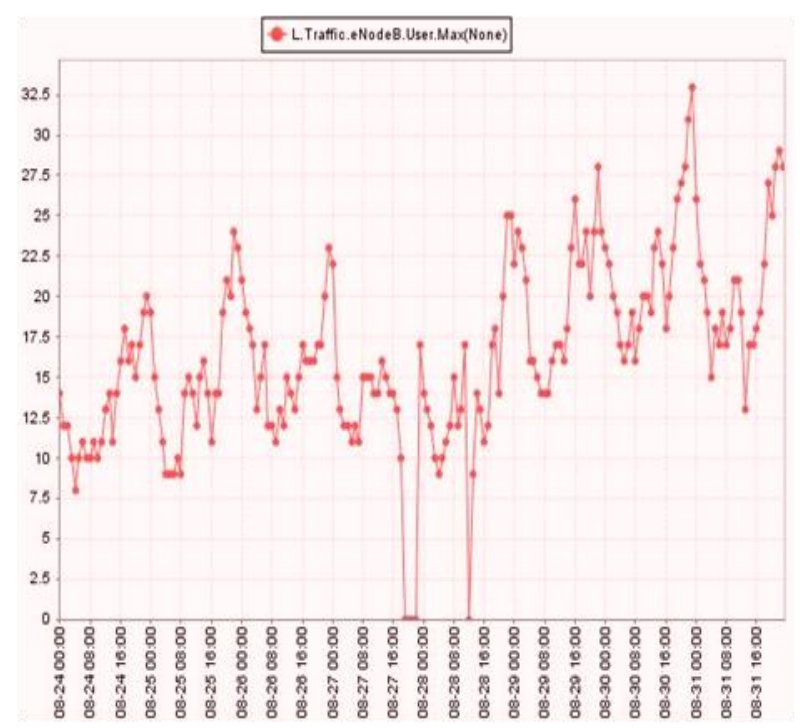

Fig 11 - Connected users with the BTS

\subsection{Discussion}

Now let's conclude the results and check how to improve spectrum more efficiently than existing structures. It has been observed in Table 1 that regardless the number of connected user's maximum throughput is $30 \mathrm{Mbps}$. If consider the total users are 17, a result obtain per user throughput of 1.764 Mbps (30 Mbps/17). In the proposed model the obtained average per user throughput (3.23 Mbps) was $83 \%$ higher than exiting models.

Although the multiple BTS test is performed with microwave as backhaul media, but, restrictions were applied to bandwidth to match copper medium. This was done due to lack of use of copper in current backhaul networks due to its limited bandwidth. However, the tests prove that copper as a backhaul can be used effectively with reduced installation costs. There are small capacity Pico BTS used in network with DSL as a backhaul media are and successfully working but only for small bandwidth of just $2 \mathrm{Mbps}$ and were used inside the Buildings like Airport, Cement industry etc.

\section{CONCLUSION}

In this paper, a solution is proposed to use copper medium as backhaul media for LTE, by using multiple BTS with lower power in an area otherwise covered by a single BTS. The proposed solution is low-cost and is particularly viable for developing countries with vast infrastructure of copper lines used for ADSL or voice services for cost. In developed countries, the copper based backhaul media can be exploit as standby in case of failure or restriction on the use of microwave or optical fiber. Along with lower cost implementation, the proposed solution helps in achieving higher network availability. Also, the paper demonstrates effective use of spectrum and it is possible to achieve higher data rates at a relatively lower cost.

It is expected that this research work on the Back haul media for LTE/4G wireless networks to enhance throughput and QoS of users at low cost \& complexity and helps make wireless cooperative networks a market realization.

\section{ACKNOWLEDGMENTS}

Special thanks to the experts who have contributed towards development of the template.

\section{REFERENCES}

[1] G. K. Venkatesan, K. Kulkarni, "Wireless backhaul for LTE - requirements, challenges and options," 2008 2nd International Symposium on Advanced Networks and Telecommunication Systems, Mumbai, 2008, pp. 1-3.

[2] C. C. Y. Yang, M. Ketcham, D. Lu and D. Kinsey, "Performance Monitoring with Predictive QoS Analysis of LTE Backhaul," 2011 International Conference on Cyber-Enabled Distributed Computing and Knowledge Discovery, Beijing, 2011, pp. 162-169.

[3] D. P. Venmani, D. Zeghlache and Y. Gourhant, "Demystifying Link Congestion in 4G-LTE Backhaul Using OpenFlow," 2012 5th International Conference on New Technologies, Mobility and Security (NTMS), Istanbul, 2012, pp. 1-8.

[4] A. Checko, L. Ellegaard and M. Berger, "Capacity planning for Carrier Ethernet LTE backhaul networks," 2012 IEEE Wireless Communications and Networking Conference (WCNC), Shanghai, 2012, pp. 2741-2745.

[5] A. Ting, D. Chieng, K. H. Kwong, I. Andonovic and K. D. Wong, "Dynamic backhaul sensitive Network Selection Scheme in LTE-WiFi wireless HetNet," 2013 IEEE 24th Annual International Symposium on Personal, Indoor, and Mobile Radio Communications (PIMRC), London, 2013, pp. 3061-3065.

[6] Monica Paolini. Senza Fili. "A backhaul solution for organic small-cell growth (white paper)". 
[7] Y. Zaki, T. Weerawardane, S. Hauth, E. Wallmeier and C. Görg, "Intelligent traffic enforcement for LTE backhaul," 2013 IEEE 24th Annual International Symposium on Personal, Indoor, and Mobile Radio Communications (PIMRC), London, 2013, pp. 30773082.

[8] Mobile network backhaul, challenges and opportunities \& Markets 2011-2016 by Juniper Research Limited, church cottage house, Basingstoke, Hampshire RG21 7QW UK
[9] Ericsson Review, 2013, Non-line-of-sight microwave backhaul for small cells, available at: http://www.ericsson.com/res/thecompany/docs/publicatio ns/ericsson_review/2013/er-nlos-microwavebackhaul.pdf

[10] Pakistan Telecommunication Authority (PTA), Annual Report 2015-16, October 07, 2016. 